\title{
COMPARATIVE STUDY OF VARIOUS SUPERVISEDCLASSIFICATION METHODSFORANALYSING DEFORESTATION FACTORS
}

\author{
S.P.Rajagopalan ${ }^{1}$, C.Lalitha ${ }^{2}$ \\ ${ }^{I}$ Professor, G.K.M College of Engineering \&Technology, Chennai, TamilNadu \\ ${ }^{2}$ Research Scholar, Vels University, Chennai, TamilNadu
}

\begin{abstract}
In this paper, various supervised classification techniques are compared and the results are demonstrated. Here the classification techniques like Decision tree, Bayesian method, Neural Networks and Rule Based method are discussed with regards to the data sets given. The population, built up and agriculture are the factors that play a vital role in the development of country which directly affects economic condition. In this paper, factors such as road, population, built up development ,agriculture and industry are considered as drivers of deforestation in the study area which is located in the Erode District of TamilNadu, India.
\end{abstract}

Keywords: Supervised Classification, Decision tree, Bayesian method, Neural Network.

\section{INTRODUCTION}

There are two types of classification, supervised and unsupervised. Supervised methods classify the data which is known and observed by the user specifically. Unsupervised methods are classified unknowably. The results are obtained with the given data sets by using the WEKA. The aim of this paper is to compare the various supervised methods by using the factors such as demographic, built up, road and agriculture. The primary methods used in Data mining are Data selection, data reduction and filtration. Data mining examines and discovers various algorithms under several computational efficiency. It integrates machine learning, pattern recognition, statistics, databases, and visualization techniques into one so that the information can be extracted from the large databases.

The tasks of data mining are association rules mining, classification, prediction and cluster analysis. Generally speaking, association rule mining and classification rule mining are the most effective and efficient techniques in data mining. Classification rule mining is used for the prediction future objects whose class label is not known. Recently it has been determined that primary factor for the degradation of ecosystem is deforestation of forests.

Classification results are basis for interpretation, analysis and modelling for various environmental and socioeconomic applications. Data mining techniques can be applied for generating the class association rules for analysing the deforestation. In this paper, we applied various supervised classification techniques with our data sets.

\section{WEKA}

The Waikato Environment for Knowledge Analysis (WEKA) is a tool for machine learning algorithms which can be used for classification and clustering. In this paper, we use decision tree methods like J48 and Random forest. Bayes algorithm such as Naive Bayes and Neural Network methods like Multilayer perceptron are implemented in WEKA. We divided our data set into 10 cross validation folds and all the methods are tested and compared according to the data.

\section{CLASSIFICATION METHODS}

\subsection{Decision Tree}

Decision Tree (DT) worksaccording to the processing and deciding upon attributable data. Here attributes in DT are considered as nodes and each leaf node as a class. J48 and Random Forest were used in our experiments. It follows a recursivemethod for a given set of data.It searches the attributes as Depth-first strategy. It divides the class into several nodes and tests each node that gives the best result.It classifies the datasets invariably. This method is not suitable for finding anddid not show good results to the given datasets.Accuracy value of J48 methods is compared and shown in Table 1 and Figure1. 


\begin{tabular}{|l|c|c|c|c|c|c|}
\hline \multicolumn{7}{|c|}{ J48 CLASSIFICATION METHOD } \\
\hline & \multicolumn{2}{|c|}{1990} & \multicolumn{2}{c|}{$\mathbf{2 0 0 0}$} & \multicolumn{2}{c|}{$\mathbf{2 0 1 0}$} \\
\hline Forests & CCI & MAE & CCI & MAE & CCI & MAE \\
\hline Population & 83.95 & $9 \mathrm{E}-04$ & 96.29 & $2 \mathrm{E}-04$ & 74.07 & 0.002 \\
\hline crop & 79.01 & 0.002 & 97.28 & $2 \mathrm{E}-04$ & 80.24 & 0.002 \\
\hline Built up & 78.02 & 0.002 & 41.48 & 0.006 & 45.18 & 0.005 \\
\hline Industry & 94.32 & 0.012 & 84.44 & 0.01 & 78.76 & 0.011 \\
\hline Road & 69.87 & 0.002 & 92.83 & $4 \mathrm{E}-04$ & 78.51 & 0.001 \\
\hline
\end{tabular}

Random forest is also called regression trees that induces the data from bootstrap samples of the training data.It uses random feature selection by induction process. Itis comparatively gives better result than CART and C4.5.

It shows a better performance, after modelling the result. The disadvantages of DT are focus on continues attributes,

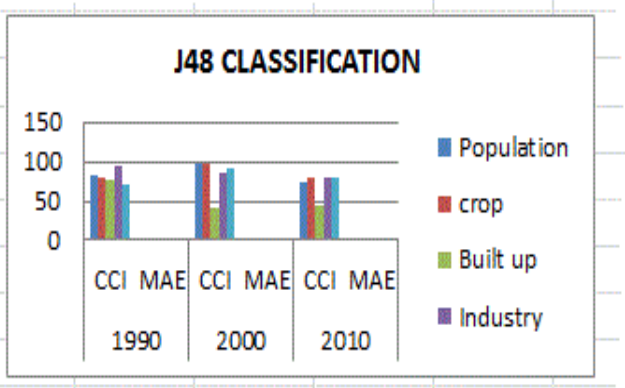

computational efficiently with growing tree size. According to comparison provided for different classification methods in emotion recognition, Random Forest is the best classifier method on that group with 5 attributes and the results are compared and shown in Table 2 and Figure 2.

\begin{tabular}{|l|c|c|c|c|c|c|}
\hline \multicolumn{5}{|c|}{ RANDOM FOREST CLASSIFICATION METHOD } \\
\hline & \multicolumn{2}{|c|}{1990} & \multicolumn{2}{c|}{2000} & \multicolumn{2}{c|}{2010} \\
\hline Forest & CCI & MAE & CCI & MAE & CCI & MAE \\
\hline Population & 99 & 0.002 & 99.01 & 0.002 & 99.5 & 0.002 \\
\hline crop & 99.75 & 0.002 & 99.5 & 0.003 & 100 & 0.003 \\
\hline Built up & 99.25 & 0.003 & 99.25 & 0.003 & 99.75 & 0.002 \\
\hline Industry & 99.75 & 0.004 & 98.51 & 0.004 & 98.51 & 0.004 \\
\hline Road & 99.25 & 0.002 & 99.5 & 0.002 & 97.03 & 0.002 \\
\hline
\end{tabular}

\subsection{Artificial Neural Networks}

\subsubsection{MLP}

Artificial Neural Network (ANN) is the common classification methods in data mining. Neural Network based classifiers, Multi Layer Perceptron (MLP) and Radial

\begin{tabular}{|l|c|c|c|c|c|c|}
\multicolumn{5}{|c|}{ MULTILAYER PERCEPTRON CLASSIFICATION METHOD } \\
\hline & \multicolumn{2}{|c|}{1990} & \multicolumn{2}{c|}{$\mathbf{2 0 0 0}$} & \multicolumn{2}{c|}{$\mathbf{2 0 1 0}$} \\
\hline Forests & MAE & C.COEFF & MAE & C.COEFF & MAE & C.COEFF \\
\hline Population & 22347 & 0.228 & 2864.9 & 0.3141 & 11191 & 0.2381 \\
\hline crop & 2417 & 0.292 & 15707 & 0.2553 & 21556 & 0.1219 \\
\hline Built up & 68.017 & 0.1542 & 113.16 & 0.379 & 145.75 & 0.3932 \\
\hline Industry & 2.21 & 0.1388 & 3.187 & 0.1746 & 8.3707 & 0.0617 \\
\hline Road & 68.017 & 0.1542 & 355.67 & 0.1538 & 349.82 & 0.1307 \\
\hline
\end{tabular}

\subsubsection{RBF}

$\mathrm{RBF}$ is another type on ANN. The input is linear and the output is nonlinear. Here it hides several non sequential values because of random input. At first it shows a good
Base Function (RBF)were used in this work. MLP is a feed forward network that makes a model to map input data to output data. Hidden layer in MLP can include various layers between input and output. It classifies 3 factors gradually. The accuracy result of MLP is shown in Table 3 and Figure 3.

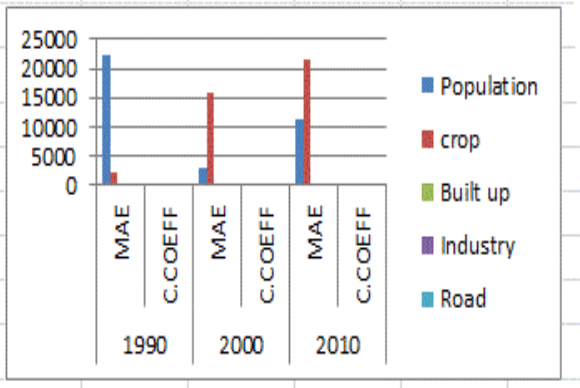

result for the year 1990 and 2010, but not for 2000.So that this algorithm is not suitable for the given datasets. The RBF networks are divided in two feed-forward layer is shown in Table 4 and Figure 4. 


\begin{tabular}{|l|c|c|c|c|c|c|}
\hline & \multicolumn{2}{|c|}{1990} & \multicolumn{2}{c|}{2000} & \multicolumn{2}{c|}{2010} \\
\hline Forest & CCI & MAE & CCI & MAE & CCI & MAE \\
\hline Population & 83.95 & $9 \mathrm{E}-04$ & 96.29 & $2 \mathrm{E}-04$ & 74.07 & 0.002 \\
\hline crop & 79.01 & 0.002 & 97.28 & $2 \mathrm{E}-04$ & 80.24 & 0.002 \\
\hline Built up & 78.02 & 0.002 & 41.48 & 0.006 & 45.18 & 0.005 \\
\hline Industry & 94.32 & 0.012 & 84.44 & 0.01 & 78.76 & 0.011 \\
\hline Road & 69.87 & 0.002 & 92.83 & $4 \mathrm{E}-04$ & 78.51 & 0.001 \\
\hline
\end{tabular}

\subsection{Bayesian Methods}

Bayesian methods are one of the solution for the classification methods in data mining. In our work, Naive Bayesis implemented for classification. It follows an independent feature model with strong independence assumptions. This method is applicable for the statistical data.

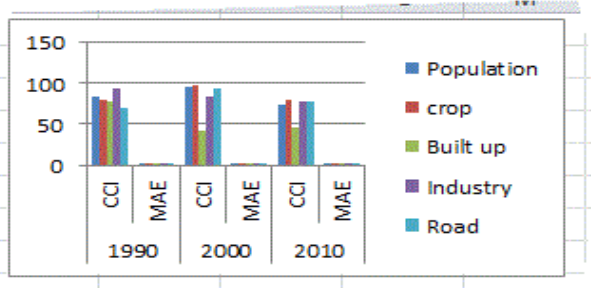

Classification is done by appropriately to the attributes Cropand built up. It classifies and shows the better performance to $10 \%$ for all the years. This method shows good results for two attributes and is displayed in Table 5 and Figure 5.

\begin{tabular}{|l|c|c|c|c|c|c|}
\hline \multicolumn{1}{|c|}{ NAIVES BAYES CLASSIFICATION METHOD } \\
\hline \multicolumn{1}{|c|}{1990} & \multicolumn{2}{c|}{2000} & \multicolumn{2}{c|}{2010} \\
\hline Forest & CCI & MAE & CCI & MAE & CCI & MAE \\
\hline Population & 7.1 & 0.005 & 2.71 & 0.005 & 13.82 & 0.006 \\
\hline crop & 14.32 & 0.007 & 16.54 & 0.007 & 29.13 & 0.006 \\
\hline Built up & 26.66 & 0.006 & 27.4 & 0.006 & 32.59 & 0.005 \\
\hline Industry & 93.82 & 0.021 & 84.69 & 0.012 & 83.7 & 0.006 \\
\hline Road & 0.49 & 0.005 & 0.988 & 0.005 & 93.08 & 0.005 \\
\hline
\end{tabular}

\subsection{Rule Based Classification}

Decision trees can be translated into a set of rules by creating a separate rule for each path from the root to a leaf in the tree. However, rules can also be directly induced from training data using a variety of rule-based algorithms. Classification accuracy of rule learning algorithms can be improved by combining features using the background knowledge of the user automatic feature construction algorithms.

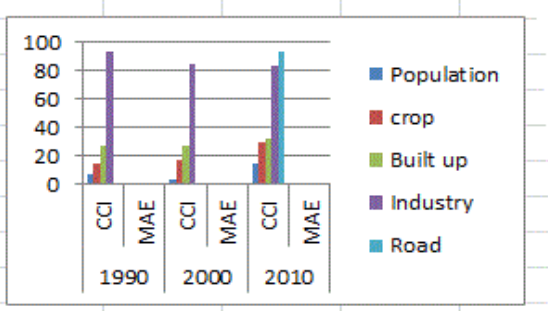

\subsubsection{R Algorithm}

One Rule is a simple accurate, classification algorithm that generates one rule for each predictor in the data and then selects the rule with the smallest total error as its "one rule". It is the simplest rule-based classification learning algorithm for discrete attributes. It shows a gradual increase in the years 1990 and 2000 for all the attributes but suddenly no changes for the year 2010 because OneR produces rules only slightly less accurate .The result of this method is shown in Table 6 and Figure 6.

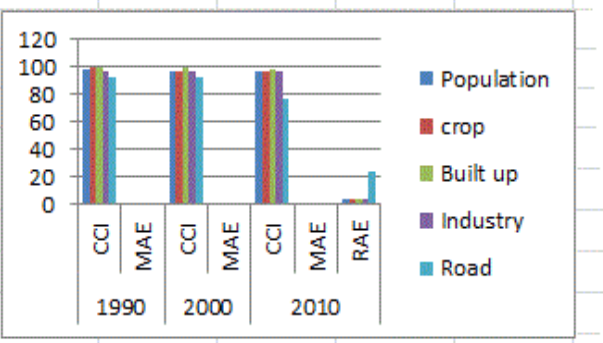

spanning forest and reflects the same result for all the three decades, is in Table 7 and Figure 7.

\subsubsection{Prism}

Prism is a greedy algorithm that finds a minimum spanning tree for a connected weighted undirected graph. This means it finds a subset of the edges that forms a tree that includes every vertex, where the total weight of all the edges in the tree is minimized. It can also be used to find the minimum

\begin{tabular}{|l|c|c|c|c|c|c|c|}
\multicolumn{7}{|c|}{ ONER CLASSIFICATION METHOD } \\
\hline & \multicolumn{2}{|c|}{1990} & \multicolumn{2}{c|}{2000} & \multicolumn{3}{c|}{$\mathbf{2 0 1 0}$} \\
\hline Forests & CCI & MAE & CCI & MAE & CCI & MAE & RAE \\
\hline Population & 98.02 & 0.004 & 96.29 & 0 & 96.29 & 0 & 3.73 \\
\hline crop & 99.75 & 0 & 96.79 & 0 & 96.79 & 0 & 3.35 \\
\hline Built up & 100 & 0 & 99.25 & 0 & 98.51 & 0 & 3.15 \\
\hline Industry & 97.28 & 0 & 96.79 & 0 & 96.79 & 0 & 3.2 \\
\hline Road & 92.96 & 0 & 92.83 & 0 & 77.03 & 0.001 & 23.02 \\
\hline
\end{tabular}




PRISM CLASSIFICATION METHOD
\begin{tabular}{|l|c|c|c|c|c|c|}
\hline \multicolumn{2}{|c|}{1990} & \multicolumn{2}{c|}{2000} & \multicolumn{2}{c|}{2010} \\
\hline Forest & CCI & MAE & CCI & MAE & CCI & MAE \\
\hline Population & 99.5 & 0 & 99.5 & 0 & 99.5 & 0 \\
\hline crop & 100 & 0 & 100 & 0 & 100 & 0 \\
\hline Built up & 100 & 0 & 100 & 0 & 100 & 0 \\
\hline Industry & 100 & 0 & 100 & 0 & 100 & 0 \\
\hline Road & 99.25 & 0 & 99.75 & 0 & 97.53 & 0 \\
\hline
\end{tabular}

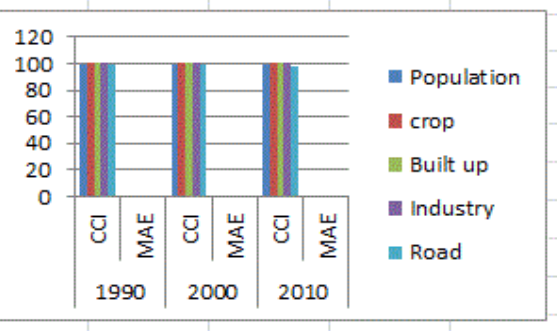

\section{COMPARATIVE RESULTS}

The Machine learning techniques such as Naïve Bayes, Bayes network, J48, Random Forest, Multi Layer Perceptron (MLP) and Radial Base Function (RBF) , one R, PRISM were used for simulation. Here we split our original dataset of 405 samples into $66 \%$ for training purpose and remaining $34 \%$ for testing purpose. Weka incorporates k-fold crossvalidation, in which the original sample is randomly partitioned into $\mathrm{k}$ subsamples. The cross-validation process

\begin{tabular}{|l|c|c|c|c|c|c|c|}
\hline \multicolumn{7}{|c|}{ Comparative study -1990 } \\
\hline Forest & J48 & RFOREST & MLP & RBF & NAIVES & ONER & PRISM \\
\hline Population & 83.95 & 99.00 & 0.228 & 83.95 & 7.1 & 98.02 & 99.5 \\
\hline crop & 79.01 & 99.75 & 0.292 & 79.01 & 14.32 & 99.75 & 100 \\
\hline Built up & 78.02 & 99.25 & 0.154 & 78.02 & 26.66 & 100 & 100 \\
\hline Industry & 94.32 & 99.75 & 0.139 & 94.32 & 93.82 & 97.28 & 100 \\
\hline Road & 69.87 & 99.25 & 0.154 & 69.87 & 0.49 & 92.96 & 99.25 \\
\hline
\end{tabular}

is then repeated into several subsamples .Here ,we have used 10 -fold cross validation .Kappa gives a numerical rating of 1 to 1 scale, where 1 is perfect agreement, 0 implies expected by chance, and negative values indicate agreement lesser than chance, Comparatively PRISM gives best result and shows the degradation of forest. The correctly classified Instances of various years are shown in Table8,9,10and the accuracy rates are displayed in Figure 8,9,10.

Fig. 8-1990

\begin{tabular}{|l|c|c|c|c|c|c|c|}
\hline \multicolumn{7}{|c|}{ Comparative study-2000 } \\
\hline Forests & J48 & RFOREST & MLP & RBF & NAIVES & ONER & PRISM \\
\hline Population & 96.29 & 99.01 & 0.314 & 96.29 & 2.71 & 96.29 & 99.5 \\
\hline crop & 97.28 & 99.50 & 0.255 & 97.28 & 16.54 & 96.79 & 100 \\
\hline Built up & 41.48 & 99.25 & 0.379 & 41.48 & 27.4 & 99.25 & 100 \\
\hline Industry & 84.44 & 98.51 & 0.175 & 84.44 & 84.69 & 96.79 & 100 \\
\hline Road & 92.83 & 99.50 & 0.154 & 92.83 & 0.988 & 92.83 & 99.75 \\
\hline
\end{tabular}

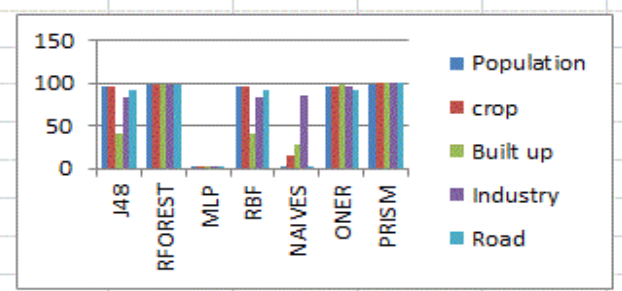

Fig.9-2000

\begin{tabular}{|l|c|c|c|c|l|c|c|}
\hline \multicolumn{7}{|c|}{ Comparative study -2010 } \\
\hline Forests & J48 & RFOREST & MLP & RBF & NAIVES & ONER & PRISM \\
\hline Population & 74.07 & 99.50 & 0.238 & 74.07 & 13.82 & 96.29 & 99.5 \\
\hline crop & 80.24 & 100 & 0.122 & 80.24 & 29.13 & 96.79 & 100 \\
\hline Built up & 45.18 & 99.75 & 0.393 & 45.18 & 32.59 & 98.51 & 100 \\
\hline Industry & 78.76 & 98.51 & 0.062 & 78.76 & 83.7 & 96.79 & 100 \\
\hline Road & 78.51 & 97.03 & 0.131 & 78.51 & 93.08 & 77.03 & 97.53 \\
\hline
\end{tabular}

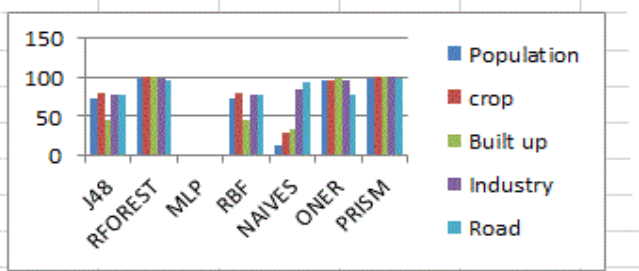

Fig. 10-2010

\section{CONCLUSIONS AND FUTURE WORK}

In this paper, four classifiers including Neural Network, Naïve Bayesian, Rule based, Decision tree were tested to determine the deforestation from the dataset of demographic factors. All the result were classified as $1,-1$ or 0 . There are many different mining and classification algorithms, and parameter settings in each algorithm. Experimental results in this paper are based on the default settings. Extensive experiments with different settings are applicable in WEKA. J48 is very simple classifier to make a decision tree, but it gives the invariable result in the experiment. Naïve Bayesian 
classifier also showed good result only for two attributes(Crop and Built up), but RBF classified another two attributes(Industry and Road) properly. Rule Based classifiers such as OneR and PRISM also showed good result than compared with J48 or Naïves Bayesian classifier. From this experiment, we can find that a simple Random Forest classifier can provide best classification result for deforestation(except one attribute).It is planned to incorporate other techniques like different ways of feature selection, classification using ontology.

\section{REFERENCES}

[1] PeimanMamaniBarnaghi+,VahidAlizadehSahzabi and Azuraliza Abu Bakar "A Comparative Study for Various Methods of Classification”, 2012 International Conference on Information and Computer Networks (ICICN 2012).

[2] LinlinXu, Jonathan Li, Alexander Brenning“ $A$ comparative study of different classification techniques for marine oil spill identification using RADARSAT-1 imagery", Remote Sensing of Environment 141 (2014) 14-23.

[3] .Elaine Astrand1, Pierre Enel2, Guilhem Ibos1, Peter Ford Dominey2, Pierre Baraduc1,Suliann Ben Hamed1* "Comparison of Classifiers for Decoding Sensory and Cognitive Information from Prefrontal Neuronal Populations ",PLOS ONE । www.plosone.org.

[4] S. B. Kotsiantis "Supervised Machine Learning: A Review of Classification Techniques"Informatica31 (2007) 249-268 249.

[5] Archana Chaudhary1, Savita Kolhe2 \& Raj Kamal3"Machine Learning Classification Techniques: A Comparative Study ",International Journal on Advanced Computer Theory and Engineering (IJACTE) ISSN ISSN (Print) : 2319 2526, Volume-2, Issue-4, 2013.

[6] Haowen You1 and George Rumbe2 "Comparative Study of Classification Techniques on Breast Cancer FNA Biopsy Data" International Journal of ArtificialIntelligence and Interactive Multimedia, Vol. 1, N $\mathrm{N}^{\mathrm{o}} 3$.

[7] PeimanMamaniBarnaghi+,VahidAlizadehSahzabi and Azuraliza Abu Bakar "A Comparative Study for Various Methods of Classification" 2012 International Conference on Information and Computer Networks (ICICN 2012) IPCSIT vol. 27 (2012) @ (2012) IACSIT Press, Singapore

[8] Gaya Buddhinathand Damien Derry "A Simple Enhancement to One Rule Classification".

[9] SeongwookYoun and Dennis McLeod "A Comparative Study for Email Classification"

[10] K.Saritha1, S. Jyothi2 \& K. R. Manjula3 "Class association rule mining for analyzing deforestation factors" International Journal of Civil, Structural,Environmental and Infrastructure Engineering Research and Development (IJCSEIERD) ISSN(P): 2249-6866; ISSN(E): 22497978 Vol. 3, Issue 5, Dec 2013, 237-248
[11] K.R.Manjula ,Prof. S. Jyothi, S.Anand Kumar Varma "Analysing the factors of deforestation using GIS "www.geospatialworld.net/paper/application/Article View.aspx ?aid $=30273$ 